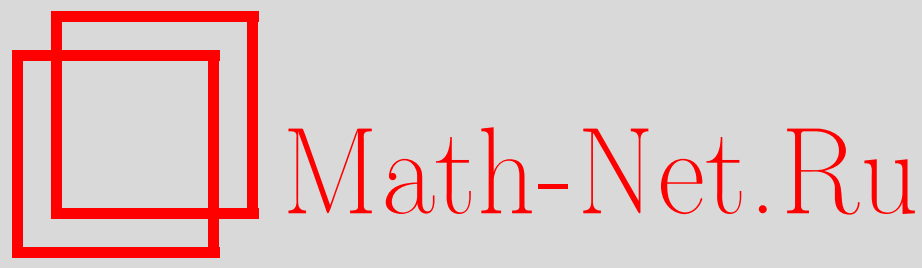

Н. В. Антонов, П. И. Какинь, Скейлинг в эрозии ландшафтов: ренормгрупповой анализ бесконечнозарядной модели, ТМФ, 2017, том 190, номер 2, 226-238

DOI: https://doi.org/10.4213/tmf9147

Использование Общероссийского математического портала Math-Net.Ru подразумевает, что вы прочитали и согласны с пользовательским соглашением http://www.mathnet.ru/rus/agreement

Параметры загрузки:

IP: 18.207 .199 .55

26 апреля 2023 г., 15:51:12

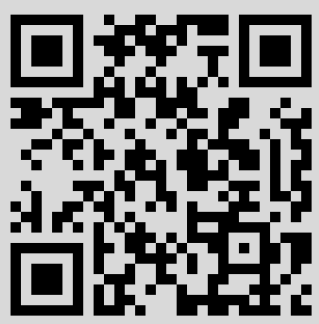




\title{
ФИЗИКА
}

Том 190, № 2

февраль, 2017

\section{СКЕЙЛИНГ В ЭРОЗИИ ЛАНДШАФТОВ: РЕНОРМГРУППОВОЙ АНАЛИЗ БЕСКОНЕЧНОЗАРЯДНОЙ МОДЕЛИ}

\begin{abstract}
Применение стандартной квантово-полевой ренормгруппы к модели эрозии ландшафтов, разработанной Пастор-Саторрасом и Ротманом, приводит к неожиданным результатам: модель оказывается мультипликативно-перенормируемой, только когда она содержит бесконечное число констант связи, т.е. соответствующие уравнения ренормгруппы содержат бесконечное число $\beta$-функций. Показано, что тем не менее однопетлевой контрчлен удается формально выразить через известную функцию $V(h)$, входящую в исходное стохастическое уравнение, и ее производные по полю $h$ высоты профиля. Из разложения этой функции в ряд Тейлора можно получить весь бесконечный набор однопетлевых перенормировочных констант, $\beta$-функций и аномальных размерностей. Вместо отдельных неподвижных точек возникает их двухмерная поверхность, которая, весьма вероятно, содержит инфракрасно-притягивающие области. В этом случае модель демонстрирует скейлинговое поведение в инфракрасном диапазоне. Соответствующие критические экспоненты оказываются неуниверсальными, поскольку они зависят от координат неподвижной точки на поверхности, но удовлетворяют определенным точным соотношениям.
\end{abstract}

Ключевые слова: турбулентность, критическое поведение, скейлинг, ренормализационная группа.

DOI: $10.4213 / \operatorname{tmf} 9147$

\section{1. ВВЕДЕНИЕ И ОПИСАНИЕ МОДЕЛИ}

Эрозия ландшафтов, происходящая под влиянием воздушных и водных течений, привлекала постоянный интерес исследователей на протяжении последних десятилетий, как и связанные с ней задачи, например гранулярные потоки (см. работы [1]-[18] и приведенную в них литературу). Разумеется, данная проблематика

Авторы благодарны Санкт-Петербургскому государственному университету за финансовую поддержку (исследовательский грант № 11.38.185.2014). П. И. Какинь благодарит РФФИ за финансовую поддержку (грант № 16-32-00086.)

* Санкт-Петербургский государственный университет, Санкт-Петербург, Россия. E-mail: n.antonov@spbu.ru,p.kakin@spbu.ru 
затрагивает широкий диапазон физических явлений; существуют разные точки зрения на лежащие в основе этих задач динамические модели [4]-[17]. Тем не менее можно надеяться, что по аналогии с критическими явлениями универсальные свойства эрозии ландшафтов (экспоненты в скейлинговых законах) могут быть описаны в рамках сравнительно простых полуфеноменологических моделей, построенных на основе соображений размерности и симметрии (см., например, обсуждение в работах [14], [15] и литературу в них).

Схожая ситуация наблюдается и в связанных с упомянутыми выше задачах кинетического огрубления поверхностей или границ раздела, описываемых хорошо известной моделью Кардара-Паризи-Жанга [19] и ее модификациями [20]-[22]. Другой пример - задачи самоорганизующейся критичности, которая в непрерывном случае описывается стохастической моделью Хуа-Кардара [23] и ее модификациями [24], [25]. В работах [14], [15] была предложена схожая модель эрозии поверхности с заданным средним наклоном. Начнем с описания этой модели.

Пусть $\mathbf{n}$ - постоянный единичный вектор, определяющий выбранное направление (направление наклона) и, таким образом, задающий внутреннюю анизотропию модели. Тогда любой вектор можно разложить на компоненты, ортогональные и параллельные вектору $\mathbf{n}$. В частности, для $d$-мерной горизонтальной координаты $\mathbf{x}$ имеем $\mathbf{x}=\mathbf{x}_{\perp}+\mathbf{n} x_{\|}$, где $\mathbf{x}_{\perp} \cdot \mathbf{n}=0$. В дальнейшем мы будем обозначать производную в полном $d$-мерном х-пространстве как $\partial=\partial / \partial x_{i}$, где $i=1, \ldots, d$, а производную в подпространстве, ортогональном к $\mathbf{n},-$ как $\partial_{\perp}=\partial / \partial x_{\perp i}$, где $i=1, \ldots, d-1$. Тогда производная вдоль параллельного направления записывается как $\partial_{\|}=\mathbf{n} \cdot \partial$.

Стохастическое дифференциальное уравнение для высоты профиля, т. е. для поля скорости $h(x)=h(t, \mathbf{x})$, предложенное в работах [14], [15], принимает вид

$$
\partial_{t} h=\nu_{\perp} \partial_{\perp}^{2} h+\nu_{\|} \partial_{\|}^{2} h+\partial_{\|}^{2} V(h)+f .
$$

Здесь $\partial_{t}=\partial / \partial t, \nu_{\|}$и $\nu_{\perp}-$ топографические коэффициенты диффузии, $V(h)$ - некоторая функция, которая зависит только от поля $h(x)$ (и не зависит от его производных), и $f(x)$ - гауссов случайный шум с нулевым средним и заданной парной корреляционной функцией

$$
\left\langle f(x) f\left(x^{\prime}\right)\right\rangle=D \delta\left(t-t^{\prime}\right) \delta^{(d)}\left(\mathbf{x}-\mathbf{x}^{\prime}\right)
$$

с некоторой положительной амплитудой $D$. Детальное обсуждение вывода модели (1.1), (1.2) и ее связи с другими моделям эрозии и самоорганизующейся критичности можно найти в работах [14], [15].

Функция $V(h)$ понимается как ряд по степеням $h$. В работах [14], [15] она полагалась нечетной по $h$, и это было продиктовано симметрией $h, f \rightarrow-h,-f$; еще одна симметрия модели - это $x_{\|} \rightarrow-x_{\|}$. Авторы работ [14], [15] обрезали ряд Тейлора $V(h)$, оставив ведущий член порядка $h^{3}$ (член, линейный по $h$, выписан в (1.1) отдельно), и затем применили к получившейся модели динамическую ренормгруппу (РГ) Вильсона и разложение по $4-d$, т. е. по отклонению размерности $d$ от ее предполагаемого верхнего критического значения $d=4$. Они установили наличие инфракрасной (ИК) притягивающей неподвижной точки в ведущем однопетелевом порядке и вычислили соответствующие критические индексы (грубость), которые, как оказалось, хорошо согласуются с экспериментальными данными, полученными из измерений морского дна.

В настоящей работе мы применяем стандартную теоретическую РГ к модели из работ [14], [15] и получаем совершенно другие результаты. План работы следующий. 
В разделе 2 мы представляем квантово-полевую формулировку стохастической задачи $(1.1),(1.2)$ для произвольной (не обязательно нечетной) полномасштабной (не обрезанной) функции $V(h)$.

В разделе 3 обсуждается ультрафиолетовая (УФ) расходимость и процедура ренормировки возникающей теории поля. Мы показываем, что верхнее критическое значение на самом деле есть $d=2$. Это приводит к значительным изменениям в РГанализе модели. А именно, оказывается, что нельзя отбрасывать вклады старших порядков разложения Тейлора функции $V(h)$, так как они неизбежно возникают как контрчлены при правильной процедуре ренормировки. Другими словами, любая обрезанная модель не является мультипликативно-ренормируемой. Это означает, что правильно построенная ренормируемая модель обязательно содержит бесконечно много констант связи, а соответствующие РГ-уравнения содержат бесконечно много $\beta$-функций. Это также означает, что РГ-анализ, проведенный в [14], [15] для обрезанной модели, не является последовательным и его нельзя считать надежным. Мы также выписываем соответствующее ренормированное действие, ренормировочные соотношения для полей и параметров, РГ-уравнения и РГ-функции ( $\beta$-функции и аномальные размерности).

В разделе 4 мы в явном виде проводим процедуру ренормировки в однопетлевом приближении. Ключевой момент состоит в том, что, несмотря на наличие бесконечного числа констант связи, однопетлевой контрчлен можно выписать формально, выразив его через функцию $V(h)$ и ее производные. Ее разложение в ряд Тейлора позволяет получить весь бесконечный набор однопетлевых ренормировочных констант и, таким образом, всех $\beta$-функций и аномальных размерностей. Для получения этого результата мы используем функциональный метод, примененный ранее А.Н. Васильевым и одним из авторов в работе [27] к изотропной модели поверхностного огрубления, предложенной в [26] в качестве возможной модификации уравнения Кардара-Паризи-Жанга (см. также статьи [28], [29]).

В разделе 5 мы анализируем аттракторы полученных РГ-уравнений в бесконечномерном пространстве констант связи. Оказывается, что вместо набора неподвижных точек (что типично для большинства моделей со многими константами связи) имеется двумерная поверхность неподвижных точек. Для нечетной функции $V(h)$, т. е. для модели из работ [14], [15], она переходит в кривую. Весьма вероятно, что эта поверхность содержит ИК-притягивающую область (области). В этом случае модель проявляет скейлинговое поведение в ИК-диапазоне. Соответствующие критические индексы оказываются неуниверсальными из-за того, что они зависят от координат неподвижной точки на поверхности (кривой), но удовлетворяют определенным точным соотношениям.

Возможность сравнения с экспериментом и остающиеся проблемы кратко обсуждаются в разделе 6 .

\section{2. КВАНТОВО-ПОЛЕВАЯ ФОРМУЛИРОВКА МОДЕЛИ}

Согласно общим утверждениям (см., например, монографии [30], [31] и литературу в них) стохастическая задача (1.1), (1.2) эквивалентна квантово-полевой модели двух полей $\Phi=\left\{h, h^{\prime}\right\}$ с функционалом действия

$$
\mathcal{S}(\Phi)=h^{\prime} h^{\prime}+h^{\prime}\left\{-\partial_{t} h+\nu_{0 \perp} \partial_{\perp 0}^{2} h+\nu_{\| 0} \partial_{\|}^{2} h+\partial_{\|}^{2} \sum_{n=2}^{\infty} \frac{\lambda_{n 0} h^{n}}{n !}\right\}
$$


(где $D_{0}$ и другие коэффициенты при $h^{\prime} h^{\prime}$ убираются путем изменения значений $\lambda_{n 0}$ ). Здесь и далее подразумеваются все необходимые интегрирования по $x=(t, \mathbf{x})$ и суммирования по повторяющимся тензорным индексам, например

$$
h^{\prime} h^{\prime}=\int d t \int d \mathbf{x} h^{\prime}(t, \mathbf{x}) h^{\prime}(t, \mathbf{x}) .
$$

Индекс 0 указывает, что параметры в (2.1) еще не ренормированы (затравочные).

Квантово-полевая формулировка означает, что корреляционные функции и функции отклика стохастической задачи (1.1), (1.2) могут быть соотнесены с различными функциями Грина квантово-полевой модели с действием (2.1). Иными словами, они представляются функциональными средними по полному набору полей $\Phi=\left\{h, h^{\prime}\right\}$ с весом $\exp \mathcal{S}(\Phi)$.

\section{3. УФ-РАСХОДИМОСТИ И РЕНОРМИРОВКА}

Для исследования УФ-расходимостей мы используем анализ канонических размерностей (см., например, монографии [30], [31]). Обычно динамические модели типа (2.1) имеют два масштаба и их размерности описываются двумя числами: частотной размерностью $d_{F}^{\omega}$ и импульсной размерностью $d_{F}^{k}$. Они полностью определяют каноническую размерность величины $F$ (поля или параметра) и определяются так, чтобы $[F] \sim[T]^{-d_{F}^{\omega}}[L]^{-d_{F}^{k}}$, где $L-$ типичный масштаб длины и $T-$ временной масштаб (см., например, гл. 5 в [31]). Для рассматриваемой задачи, однако, из-за анизотропии возникают два независимых импульсных масштаба, соответствующих перпендикулярному и параллельному к вектору $\mathbf{n}$ направлениям, поэтому нам потребуется более детальное определение. А именно, нужно ввести два независимых импульсных канонических масштаба $d_{F}^{\perp}$ и $d_{F}^{\|}$так, чтобы было выполнено соотношение

$$
[F] \sim[T]^{-d_{F}^{\omega}}\left[L_{\perp}\right]^{-d_{F}^{\perp}}\left[L_{\|}\right]^{-d_{F}^{\|}}
$$

где $L_{\perp}$ и $L_{\|}-$независимые масштабы длины в соответствующих подпространствах. Очевидны условия нормировки: $d_{k_{\perp}}^{\perp}=-d_{\mathbf{x}_{\perp}}^{\perp}=1, d_{k_{\perp}}^{\|}=-d_{\mathbf{x}_{\perp}}^{\|}=0, d_{k_{\perp}}^{\omega}=d_{k_{\|}}^{\omega}=0$, $d_{\omega}^{\omega}=-d_{t}^{\omega}=1$ и т. д.; остальные размерности определяются из требования, чтобы каждый член функционала действия (2.1) был безразмерным (по отношению ко всем трем размерностям по отдельности). Исходную импульсную размерность можно найти из соотношения $d_{F}^{k}=d_{F}^{\perp}+d_{F}^{\|}$. Тогда, основываясь на $d_{F}^{k}$ и $d_{F}^{\omega}$, можно ввести полную каноническую размерность $d_{F}=d_{F}^{k}+2 d_{F}^{\omega}=d_{F}^{\perp}+d_{F}^{\|}+2 d_{F}^{\omega}$ (в свободной теории $\left.\partial_{t} \propto \partial_{\perp}^{2} \propto \partial_{\|}^{2}\right)$, которая играет ту же роль, что и привычная (импульсная) размерность в статических задачах (см. [31], гл. 5).

Канонические размерности полей и параметров в модели с действием (2.1) представлены в табл. 1. Ренормированные параметры (без нижнего индекса 0) и ренормировочная масса $\mu$ будут определены ниже.

Как видно из табл. 1, все константы взаимодействия $g_{n 0}$ одновременно становятся безразмерными при $d=2$. Это означает, что $d=2-$ верхнее критическое значение размерности в полномасштабной модели. При этом значении $d$ полная каноническая размерность поля $h$ равна нулю. Как будет показано ниже, это приводит к значительным изменениям в процедуре ренормировки. Это также означает, что 
ТАБлицА 1. Канонические размерности полей и параметров в модели (2.1).

\begin{tabular}{c|cccccccc}
\hline$F$ & $h^{\prime}$ & $h$ & $\nu_{\perp}$ & $\nu_{\|}$ & $\lambda_{n 0}$ & $g_{n 0}$ & $g_{n}$ & $\mu$ \\
\hline$d_{F}^{\omega}$ & $\frac{1}{2}$ & $-1 / 2$ & 1 & 1 & $\frac{n+1}{2}$ & 0 & 0 & 0 \\
$d_{F}^{\|}$ & $\frac{1}{2}$ & $\frac{1}{2}$ & 0 & -2 & $-\frac{n+3}{2}$ & 0 & 0 & 0 \\
$d_{F}^{\perp}$ & $\frac{d-1}{2}$ & $\frac{d-1}{2}$ & -2 & 0 & $\frac{(d-1)(1-n)}{2}$ & $\frac{(2-d)(n-1)}{2}$ & 0 & 1 \\
\hline$d_{F}$ & $\frac{2+d}{2}$ & $-\frac{2-d}{2}$ & 0 & 0 & $\frac{(2-d)(n-1)}{2}$ & $\frac{(2-d)(n-1)}{2}$ & 0 & 1 \\
\hline
\end{tabular}

УФ-расходимости в функциях Грина полномасштабной модели предстают в виде полюсов по $\varepsilon=2-d$, при этом $\varepsilon$ играет роль малого параметра в РГ-разложениях.

Полная каноническая размерность произвольной 1-неприводимой функции Грина $\Gamma=\langle\Phi \ldots \Phi\rangle_{1 \text {-ir }}$ с $\Phi=\left\{h, h^{\prime}\right\}$ в частотно-импульсном представлении дается соотношением

$$
d_{\Gamma}=d+2-d_{h} N_{h}-d_{h^{\prime}} N_{h^{\prime}}
$$

где $N_{h}, N_{h^{\prime}}$ - число соответствующих полей, входящих в функцию $\Gamma$ [31].

Полная размерность $d_{\Gamma}$ в логарифмической теории (т. е. при $\left.\varepsilon=0\right)$ - это формальный индекс УФ-расходимости: $\delta_{\Gamma}=\left.d_{\Gamma}\right|_{\varepsilon=0}$. Поверхностная УФ-расходимость, для исключения которой нужны контрчлены, может присутствовать только в тех функциях $\Gamma$, для которых $\delta_{\Gamma}-$ неотрицательное целое число. Контрчлен есть полином по частотам и импульсам степени $\delta_{\Gamma}$ (при условии, что подразумевается $\omega \propto k^{2}$ ).

Если по какой-либо причине некоторое число внешних импульсов выделяется как общий множитель во всех диаграммах данной функции Грина, то реальный индекс расходимости $\delta_{\Gamma}^{\prime}$ оказывается меньше, чем $\delta_{\Gamma}$, на соответствующее число единиц. Именно это и происходит в нашей модели: с помощью интегрирования по частям производная в вершине $h^{\prime} \partial_{\|}^{2} V(h)$ переносится на $h^{\prime}$. Это означает, что любое появление $h^{\prime}$ в какой-либо функции Г приводит к появлению внешних импульсов, и реальный индекс расходимости оказывается равным $\delta_{\Gamma}^{\prime}=\delta_{\Gamma}-2 N_{h^{\prime}}$. Более того, $h^{\prime}$ возникает в соответствующем контрчлене только в форме производной $\partial_{\|}^{2} h^{\prime}$.

Из табл. 1 и выражения (3.1) получаем

$$
\delta_{\Gamma}^{\prime}=\delta_{\Gamma}-2 N_{h^{\prime}}=4-4 N_{h^{\prime}}
$$

Достаточно рассмотреть только случай $N_{h^{\prime}}>0$, так как в динамических моделях все 1-неприводимые функции без полей отклика тождественно равны нулю (их диаграммы всегда содержат замкнутые циклы запаздывающих линий) [31].

Простой анализ выражения (3.2) показывает, что поверхностные УФ-расходимости могут присутствовать только в 1-неприводимых функциях вида $\left\langle h^{\prime} h \ldots h\right\rangle_{1 \text {-ir }}$ с контрчленами $\left(\partial_{\|}^{2} h^{\prime}\right) h^{n}$ (для любого $n \geqslant 1$ ). Действительно, все прочие контрчлены (например, $\left.h^{\prime} h^{\prime}, h^{\prime} \partial_{t} h, h^{\prime} \partial_{\perp}^{2} h\right)$ не нужны, так как соответствующие 1 -неприводимые функции конечны. 
Поскольку все члены вида $\left(\partial_{\|}^{2} h^{\prime}\right) h^{n}$ присутствуют в действии $(2.1)$, полномасштабная модель мультипликативно-ренормируема. Ренормированное действие может быть записано в форме

$$
\mathcal{S}_{\mathrm{R}}(\Phi)=h^{\prime} h^{\prime}+h^{\prime}\left\{-\partial_{t} h+Z_{\perp} \nu_{\perp} \partial_{\perp}^{2} h+Z_{\|} \nu_{\|} \partial_{\|}^{2} h+\partial_{\|}^{2} \sum_{n=2}^{\infty} \frac{Z_{n} \lambda_{n} h^{n}}{n !}\right\},
$$

где $\nu_{\perp}, \nu_{\|}$и $\lambda_{n}$ - ренормированные аналоги затравочных параметров (с нижним индексом 0). Константы ренормировки $Z_{\perp}, Z_{\|}$и $Z_{n}$ зависят только от полностью безразмерных параметров $g_{n}$ и поглощают все полюсы по $\varepsilon$. Затравочные заряды $g_{0}=\left\{g_{n 0}\right\}$ и полностью безразмерные ренормированные заряды $g=\left\{g_{n}\right\}$ $(n=2,3, \ldots)$ выражаются через затравочные параметры $\lambda_{n 0}$ и ренормированные параметры $\lambda_{n}$ следующим образом:

$$
\lambda_{n 0}=g_{n 0} \nu_{\| 0}^{(n+3) / 4} \nu_{\perp 0}^{(n-1) / 4}, \quad \lambda_{n}=g_{n} \nu_{\|}^{(n+3) / 4} \nu_{\perp}^{(n-1) / 4} \mu^{\varepsilon(n-1) / 2},
$$

где ренормировочная масса $\mu$ является добавочным параметром ренормированной теории; ее канонические размерности показаны в табл. 1.

Ренормированное действие (3.3) получается из исходного (2.1) путем ренормировки параметров (ренормировка полей $h, h^{\prime}$ не требуется)

$$
\nu_{\| 0}=\nu_{\|} Z_{\|}, \quad \nu_{\perp 0}=\nu_{\perp} Z_{\perp}, \quad g_{n 0}=\mu^{\varepsilon(n-1) / 2} g_{n} Z_{g_{n}}, \quad \lambda_{n 0}=\lambda_{n} Z_{n} .
$$

Константы ренормировки в уравнениях (3.3) и (3.5) связаны следующим образом:

$$
Z_{g_{n}}=Z_{n} Z_{\|}^{-(n+3) / 4} Z_{\perp}^{-(n-1) / 4} .
$$

Рассмотрим простой вывод РГ-уравнений [30], [31]. РГ-уравнения пишутся для перенормированных функций Грина $G_{\mathrm{R}}=\langle\Phi \ldots \Phi\rangle_{\mathrm{R}}$. В нашем случае они не отличаются от исходных (неренормированных) функций Грина $G: G\left(e_{0}, \ldots\right)=G_{\mathrm{R}}(e, \mu, \ldots)$ (так как отсутствует ренормировка полей) и поэтому также могут быть использованы для анализа критического поведения. Здесь $e_{0}=\left\{g_{n 0}, \nu_{\| 0}, \nu_{\perp 0}, \ldots\right\}-$ полный набор затравочных параметров и $e=\left\{g_{n}, \nu_{\|}, \nu_{\perp}, \ldots\right\}$ - их ренормированные эквиваленты; многоточием обозначены остальные аргументы (время, координаты, импульсы).

Обозначим через $\widetilde{\mathcal{D}}_{\mu}$ дифференциальный оператор $\left.\mu \partial_{\mu}\right|_{e_{0}}$. Если выразить его через ренормированные переменные, он примет вид

$$
\mathcal{D}_{\mathrm{RG}} \equiv \mathcal{D}_{\mu}+\sum_{n=2}^{\infty} \beta_{n} \partial_{g_{n}}-\sum_{F=\nu_{\|}, \nu_{\perp}} \gamma_{F} \mathcal{D}_{F}
$$

где $\mathcal{D}_{x} \equiv x \partial_{x}$ для любой переменной $x$. Аномальная размерность $\gamma_{F}$ для какой-либо величины $F$ определяется как

$$
\gamma_{F} \equiv \widetilde{\mathcal{D}}_{\mu} \ln Z_{F}
$$

в то время как $\beta$-функции для безразмерных констант взаимодействия $g_{n}$ выглядят следующим образом:

$$
\beta_{n} \equiv \widetilde{\mathcal{D}}_{\mu} g_{n}=g_{n}\left[-\varepsilon \frac{n-1}{2}-\gamma_{g_{n}}\right]
$$




\section{4. ОДНОПЕТЛЕВЫЕ ВЫРАЖЕНИЯ ДЛЯ КОНТРЧЛЕНА, КОНСТАНТ РЕНОРМИРОВКИ И РГ-ФУНКЦИЙ}

Обратимся к вычислению констант ренормировки $Z$ в однопетлевом приближении. Несмотря на то что полномасштабная модель содержит бесконечно много констант взаимодействия, однопетлевое выражение для контрчлена можно получить в явном виде, выразив его формально через функцию $V(h)$.

Рассмотрим разложение производящего функционала $\Gamma_{\mathrm{R}}(\Phi) 1$-неприводимой функции Грина нашей модели в $p$ петлях:

$$
\Gamma_{\mathrm{R}}(\Phi)=\sum_{p=0}^{\infty} \Gamma^{(p)}(\Phi), \quad \Gamma^{(0)}(\Phi)=S_{\mathrm{R}}(\Phi) .
$$

Беспетлевой (древесный) вклад - это просто действие, тогда как однопетлевой вклад можно найти с помощью следующего соотношения [32]:

$$
\Gamma^{(1)}(\Phi)=-\frac{1}{2} \operatorname{Tr} \ln \left(W W_{0}^{-1}\right)
$$

где $W$ - линейный оператор с ядром

$$
W(x, y)=-\frac{\delta^{2} S_{\mathrm{R}}(\Phi)}{\delta \Phi(x) \delta \Phi(y)}
$$

и $W_{0}$ - аналогичное выражение для свободной части действия; $W$ и $W_{0}$ - матрицы размера $2 \times 2$ для пары полей $\Phi=\left\{h, h^{\prime}\right\}$.

Требование, чтобы в выражении (4.1) не было УФ-расходимостей, вместе со схемой минимальных вычитаний приводит к однозначно определенным значениям для констант $Z$. Мы полагаем $Z=1$ в (4.2) в однопетлевом приближении, одновременно удерживая вклады ведущего порядка в константах взаимодействия $g_{n}$ в беспетлевом вкладе в константы $Z$; для внутренней согласованности мы полагаем $g_{n} \simeq g_{2}^{n-1}$.

Рассмотрим ряд Тейлора для функции $V(h)$ :

$$
V(h)=\sum_{n=2}^{\infty} \lambda_{n} \frac{h^{n}(x)}{n !}, \quad V_{\mathrm{R}}(h)=\sum_{n=2}^{\infty} Z_{n} \lambda_{n} \frac{h^{n}(x)}{n !} .
$$

В дальнейшем мы будем интерпретировать подобные объекты как функции единственной переменной $h(x)$, a $V^{\prime}, V^{\prime \prime}$ и т. д. - как соответствующие производные по этой переменной. В этих обозначениях матрицу $W$ (при условии, что $Z=1$ ) можно формально записать как

$$
W=\left(\begin{array}{cc}
-\partial_{\|}^{2} h^{\prime} \cdot V^{\prime \prime} & L^{\mathrm{T}} \\
L & -2
\end{array}\right),
$$

где $L \equiv \partial_{t}-\nu_{\|} \partial_{\|}^{2}-\nu_{\perp} \partial_{\perp}^{2}-\partial_{\|}^{2} V^{\prime}$ и $L^{\mathrm{T}} \equiv-\partial_{t}-\nu_{\|} \partial_{\|}^{2}-\nu_{\perp} \partial_{\perp}^{2}-V^{\prime} \partial_{\|}^{2}-$ транспонированный оператор.

Чтобы найти константы $Z$, нам потребуется только расходящаяся часть выражения (4.1), которая, как было установлено ранее, имеет вид

$$
\int d x \partial^{2} h^{\prime}(x) R(h(x))
$$


где функция $R(h)$ аналогична $V(h)$. Это значит, что след логарифма в $(4.2)$ с матрицей (4.5) нужно вычислить только в первом порядке по ее $h h$-элементу $-\partial_{\|}^{2} h^{\prime} \cdot V^{\prime \prime}$. Это можно сделать с помощью хорошо известной формулы $\delta(\operatorname{Tr} \ln K)=\operatorname{Tr}\left(K^{-1} \delta K\right)$, справедливой для любой вариации $\delta K$. Варьируя только $h h$-элемент матрицы $W$, получаем

$$
\int d x \partial^{2} h^{\prime}(x) R(h(x)) \simeq-\operatorname{Tr}\left[D_{h h} V^{\prime \prime} \partial_{\|}^{2} h^{\prime}\right]=-\int d x D^{(h h)}(x, x) V^{\prime \prime}(h(x)) \partial_{\|}^{2} h^{\prime}(x),
$$

где $D_{h h}=\left(W^{-1}\right)_{h h}$ при $h^{\prime}=0$. По определению $D^{(h h)}-$ обыкновенный пропагатор $\langle h h\rangle$ модели (3.3) с $Z=1$, в котором вместо $\nu_{\|} \partial_{\|}^{2}+\nu_{\perp} \partial_{\perp}^{2}$ стоит $\nu_{\|} \partial_{\|}^{2}+\nu_{\perp} \partial_{\perp}^{2}+\partial_{\|}^{2} V^{\prime}$.

Есть еще одно соображение, которое следует учесть. После того как $\partial_{\|}^{2}$ превращается во внешний множитель для $h^{\prime}$, в контрчлене остается только логарифмически расходящаяся часть. Это означает, что при подсчете расходящейся части какой-либо диаграммы все внешние импульсы можно положить равными нулю (ИКрегуляризация обеспечивается обрезанием). В свою очередь это приводит к тому, что в (4.6) можно игнорировать неоднородность функций $\partial_{\|}^{2} h^{\prime}(x)$ и $h(x)$ (оба поля можно считать константами), когда мы отбираем полюсы по $\varepsilon$. Тогда можно легко вычислить $D^{(h h)}(x, x)$, перейдя к частотно-импульсному представлению:

$$
\begin{aligned}
D^{(h h)}(x, x) & =\iint \frac{d \omega d \mathbf{k}}{(2 \pi)^{d+1}} \frac{2}{\omega^{2}+\left(\nu_{\|} k_{\|}^{2}+\nu_{\perp} k_{\perp}^{2}+k_{\|}^{2} V^{\prime}\right)^{2}}= \\
& =\frac{S_{d}}{(2 \pi)^{d}} \frac{\mu^{-\varepsilon}}{\varepsilon} \frac{1}{\sqrt{\nu_{\perp}\left(\nu_{\|}+V^{\prime}\right)}}+\cdots,
\end{aligned}
$$

где многоточие означает УФ-конечную часть. Подставляя выражения (4.6) и (4.7) в (4.2), получаем следующее выражение для расходящейся части $\Gamma_{1}(\Phi)$ с необходимой точностью:

$$
\Gamma_{1}(\Phi)=\frac{S_{d}}{2(2 \pi)^{d}} \frac{\mu^{-\varepsilon}}{\varepsilon} \int d x \frac{V^{\prime \prime}(h(x))}{\sqrt{\nu_{\perp}\left(\nu_{\|}+V^{\prime}(h(x))\right)}} \partial^{2} h^{\prime}(x) .
$$

Найти однопетлевые вклады порядка $1 / \varepsilon$ во всех константах $Z$ можно из требования, что сумма $\Gamma_{1}(\Phi)$ из (4.8) и беспетлевых вкладов в $(4.2)$ не имеет полюса по $\varepsilon$ (полюсы сокращаются).

Введем представление

$$
\frac{V^{\prime \prime}(h(x))}{\sqrt{\nu_{\perp}\left(\nu_{\|}+V^{\prime}(h(x))\right)}}=\sum_{n=0}^{\infty} \mu^{\varepsilon(n+1) / 2} \nu_{\perp}^{(n-1) / 4} \nu_{\|}^{(n+3) / 4} \frac{r_{n} h^{n}}{n !}
$$

для ряда Тейлора подынтегральной функции в (4.8). Тогда $r_{n}$ - полностью безразмерные коэффициенты, являющиеся полиномами по зарядам $g_{n}$. Объединяя описанное выше условие сокращения полюсов по $\varepsilon$ и формулы (3.4), получаем

$$
Z_{\perp}=1, \quad Z_{\|}=1-\frac{r_{1} S_{d}}{2(2 \pi)^{d} \varepsilon}+\cdots, \quad Z_{n}=1-\frac{r_{n}}{g_{n}} \frac{S_{d}}{2(2 \pi)^{d} \varepsilon}+\cdots .
$$


Оператор $\widetilde{\mathcal{D}}_{\mu}$ в $(3.9)$ принимает вид

$$
\widetilde{\mathcal{D}}_{\mu}=\sum_{n}\left(\widetilde{\mathcal{D}}_{\mu} g_{n}\right) \partial_{g_{n}}=\sum_{n} \beta_{n} \partial_{g_{n}}
$$

Поэтому, для того чтобы получить необходимую точность, достаточно использовать только первые вклады в $\beta$-функции (3.9). Это дает

$$
\widetilde{\mathcal{D}}_{\mu} \simeq-\frac{\varepsilon}{2} \mathcal{D}_{g}, \quad \mathcal{D}_{g}=\sum_{n=2}^{\infty}(n-1) g_{n} \partial_{g_{n}} .
$$

Данное соображение вместе с соотношениями (4.10), (3.6) и (3.9) приводит к следующему выражению для однопетлевых РГ-функций:

$$
\begin{gathered}
\gamma_{\|}=a \frac{\mathcal{D}_{g} r_{1}}{2}, \quad a \equiv \frac{S_{d}}{2(2 \pi)^{d}}, \\
\beta_{n}=-\varepsilon \frac{n-1}{2} g_{n}+\frac{n+3}{4} g_{n} \gamma_{\|}-\frac{a}{2}\left(\mathcal{D}_{g}-n+1\right) r_{n} .
\end{gathered}
$$

Явные выражения для первых четырех коэффициентов $r_{n}$ (первый член $r_{0}$ в $(4.9)$ не дает вклада в (4.8)) находятся из определений (4.9), (4.4), (3.4):

$$
\begin{aligned}
& r_{1}=g_{3}-\frac{1}{2} g_{2}^{2}, \quad r_{2}=g_{4}-\frac{3}{2} g_{2} g_{3}+\frac{3}{4} g_{2}^{3}, \\
& r_{3}=g_{5}-2 g_{2} g_{4}-\frac{3}{2} g_{3}^{2}+\frac{9}{2} g_{2}^{2} g_{3}-\frac{15}{8} g_{2}^{4}, \\
& r_{4}=g_{6}-\frac{5}{2} g_{2} g_{5}+\frac{15}{2} g_{2}^{2} g_{4}-5 g_{3} g_{4}+\frac{45}{4} g_{2} g_{3}^{2}-\frac{75}{4} g_{2}^{3} g_{3}+\frac{105}{16} g_{2}^{5}
\end{aligned}
$$

при подстановке в (4.12) они дают

$$
\begin{gathered}
\gamma_{\|}=\frac{a}{2}\left(2 g_{3}-g_{2}^{2}\right), \\
\beta_{2}=-\frac{\varepsilon}{2} g_{2}+a\left(-g_{4}+\frac{11}{4} g_{2} g_{3}-\frac{1}{8} g_{2}^{3}\right) \\
\beta_{3}=-\varepsilon g_{3}+a\left(-g_{5}+2 g_{2} g_{4}+3 g_{3}^{2}-\frac{21}{4} g_{2}^{2} g_{3}+\frac{15}{8} g_{2}^{4}\right) .
\end{gathered}
$$

Напомним, что мы положили $g_{n} \sim g_{2}^{n-1}$ для внутренней согласованности приближения.

\section{5. АТТРАКТОРЫ И КРИТИЧЕСКИЕ ИНДЕКСЫ}

Обратимся к полной системе (4.15) $\beta$-функций. Неподвижные точки РГ-уравнений находятся из условия $\beta_{n}\left(g_{*}\right)=0, n=2,3, \ldots$ Я Явный вид $\beta$-функций (4.15) показывает, что мы можем выбрать координаты $g_{2 *}$ и $g_{3 *}$ произвольно, тогда все $g_{n *}$ с $n \geqslant 4$ однозначно определяются через них с помощью уравнений $\beta_{k}\left(g_{*}\right)=0$ для $k \geqslant 3$. Это означает, что в бесконечномерном пространстве констант взаимодействия $g \equiv\left\{g_{n}\right\}$ РГ-уравнения (3.7) имеют двумерную поверхность неподвижных точек, параметризованную значениями $g_{2 *}$ и $g_{3 *}$. 
В общем случае анализ этих точек - непростая задача. Тем не менее согласно общему правилу [30] точка $g_{*}$ ИК-устойчива, если все действительные части собственных чисел матрицы с элементами $\omega_{n m}=\partial \beta_{n}\left(g_{*}\right) / \partial g_{m}$ строго положительны. Для этого необходимо, чтобы все диагональные элементы $\omega_{n n}$ были положительны. Из уравнения (4.13) можно получить эти элементы для всех значений $n$ :

$$
\omega_{22}=-\frac{\varepsilon}{2}+a\left[\frac{11}{4} g_{3 *}-\frac{3}{8} g_{2 *}^{2}\right], \quad \omega_{33}=-\varepsilon+a\left[6 g_{3 *}-\frac{21}{4} g_{2 *}^{2}\right],
$$

а для $n \geqslant 4$ имеем

$$
\omega_{n n}=-\varepsilon \frac{n-1}{2}+a \frac{(n+1)^{2}+2}{4} g_{3 *}-\frac{a}{8}(n(3 n+4)+3) g_{2 *}^{2} .
$$

В области $g_{3 *} \geqslant 7 g_{2 *}^{2} / 8+\varepsilon / 6$ все эти величины положительны. Конечно, это только необходимое условие, тем не менее мы можем предположить, что поверхность неподвижных точек $g_{*}$ содержит область ИК-устойчивости. Если это действительно так, модель может проявлять ИК-скейлинг с неуниверсальными критическими размерностями (т. е. с размерностями, не зависящими от параметров $g_{2 *}$ и $g_{3 *}$ ).

В динамических моделях типа (2.1) критическая размерность $\Delta_{F}$ произвольной величины $F$ (поля или параметры) задается следующими выражениями:

$$
\Delta_{F}=d_{F}^{\perp}+d_{F}^{\|} \Delta_{\|}+d_{F}^{\omega} \Delta_{\omega}+\gamma_{F}^{*}, \quad \Delta_{w}=2-\gamma_{\perp}^{*}, \quad \Delta_{\|}=1+\frac{\gamma_{\|}^{*}}{2} .
$$

В нашем случае для $F=h$ имеем $\gamma_{h}^{*}=0$ и $\gamma_{\perp}^{*}=0$ (поля и параметр $\nu_{\perp}$ не ренормируются). Из соотношений (5.1), используя табл. 1 , получаем точный результат $2 \Delta_{h}=d-1+\Delta_{\|}-\Delta_{\omega}$; из выражения (4.14) мы находим в однопетлевом приближении

$$
\Delta_{\|}=1+a \frac{2 g_{3 *}-g_{2 *}^{2}}{4}, \quad \Delta_{h}=a \frac{2 g_{3 *}-g_{2 *}^{2}}{8} .
$$

\section{6. ЗАКЛЮЧЕНИЕ}

Мы применили к модифицированной модели из работ [14], [15] стандартную квантово-полевую РГ. Оказалось, что эта модель может быть переформулирована как ренормируемая квантово-полевая модель с бесконечным числом независимых констант ренормировки (и, таким образом, с бесконечным числом констант взаимодействия). Действительно, для того чтобы построить ренормируемую модель, необходимо включить бесконечно много констант взаимодействия, вследствие чего соответствующие РГ-уравнения содержат бесконечно много $\beta$-функций. Несмотря на это, оказывается возможным получить однопетлевой контрчлен, используя метод, предложенный ранее в работе [27] для изотропной модели поверхностного огрубления. Метод дает двухмерную поверхность неподвижных точек, которая, вероятно, содержит область или области ИК-устойчивости. Действительно, результаты экспериментов (см. обсуждение в работе [15]) свидетельствуют о наличии двух широких диапазонов значений критического индекса огрубления, что может объясняться наличием двух разных областей ИК-устойчивости.

Модель, для того чтобы быть ренормируемой, должна содержать бесконечное число констант взаимодействия, поэтому "укороченные" модели типа моделей из работ [14], [15] или моделей с нечетной $V(h)$ могут не подходить для РГ-анализа. 
Наивный способ сравнения результатов для модели из [14], [15] с результатами настоящей статьи путем приравнивания к нулю соответствующих констант взаимодействия в $V(h)$ показывает, что согласия нет. Чтобы сравнить критические индексы двух моделей, нужно положить $z_{\perp}=\Delta_{\omega}, \zeta_{\perp}=\Delta_{\|}, \alpha_{\perp}=\Delta_{h}$. Очевидный подсчет показывает, что в случае $\lambda_{n}=0$ для всех $n$ кроме $n=3$ критические индексы равны $\Delta_{\omega}=2, \Delta_{\|}=1+(2-d) / 6$ и $\Delta_{h}=(2-d) / 12$. Последние два значения отличаются от полученных в [14], [15].

Для нечетной функции $V(h)$ двухмерная поверхность неподвижных точек переходит в кривую. Из соображений симметрии и из явного выражения (4.8) для контрчлена видно, что эта модель ренормируема. Нужно просто положить все нечетные константы взаимодействия $g_{2 n+1}$ и соответствующие $\beta$-функции в (4.13) равными нулю.

Если поверхность неподвижных точек действительно содержит области ИК-устойчивости, то модель проявляет скейлинговое поведение. Соответствующие критические индексы оказываются неуниверсальными, так как они зависят от координат неподвижной точки на поверхности (кривой). Тем не менее критические индексы удовлетворяют определенным точным соотношениям.

В дальнейшем было бы интересно исследовать поведение модели в случае, когда в нее включено турбулентное поле скорости (изотропный случай см. в работе [28]).

С более теоретической точки зрения хотелось бы выписать РГ-уравнения и найти неподвижные точки в терминах функции $V(h)$, чтобы вместо бесконечного числа $\beta$-функций для бесконечного числа констант взаимодействия $g_{n}$ иметь только один функционал $\beta(V)$ с одним аргументом $V(h)$ (см. обсуждение в статье [33] для общего случая). Эта работа уже ведется, но еще не завершена.

Благодарности. Авторы выражают признательность Л. Ц. Аджемяну, М. Гнатичу, Ю. Хонконену и М. Ю. Налимову за полезные обсуждения. Авторы благодарят организаторов V Международной конференции "Модели квантовой теории поля" (MQFT-2015) за возможность представить результаты данного исследования.

\section{Список литературы}

[1] M. J. Kirkby, "Hillslope process-response models based on the continuity equation", Slopes: Form and Process, ed. D. Brunsden, Institute of British Geographers, London, 1971, 15-29.

[2] A. E. Scheidegger, Theoretical Geomorphology, Springer, New York, 1991.

[3] I. Rodríguez-Iturbe, A. Rinaldo, Fractal River Basins: Chance and Self-Organization, Cambridge Univ. Press, Cambridge, 1997.

[4] A. D. Howard, G. Kerby, "Channel changes in badlands", Geol. Soc. Am. Bull., 94:6 (1983), 739-752.

[5] J. W. Kirchner, "Statistical inevitability of Horton's laws and the apparent randomness of stream channel networks", Geology, 21:7 (1993), 591-594.

[6] G. Willgoose, R. L. Bras, I. Rodríguez-Iturbe, "A coupled channel network growth and hillslope evolution model: 1. Theory", Water Resour. Res., 27:7 (1991), 1671-1684.

[7] D.S. Loewenherz, "Stability and the initiation of channelized surface drainage: A reassessment of the short wavelength limit", J. Geophys. Res.: Solid Earth, 96:B5 (1991), 8453-8464.

[8] A. D. Howard, "A detachment-limited model of drainage basin evolution", Water Resour. Res., 30:7 (1994), 2261-2285; A. D. Howard, W. E. Dietrich, M. A. Seidl, "Modeling fluvial 
erosion on regional to continental scales", J. Geophys. Res.: Solid Earth, 99:B7 (1994), 13971-13986.

[9] N. Izumi, G. Parker, "Inception of channelization and drainage basin formation: upstreamdriven theory", J. Fluid Mech., 283 (1995), 341-363.

[10] A. Giacometti, A. Maritan, J. R. Banavar, "Continuum model for river networks", Phys. Rev. Lett., 75:3 (1995), 577-580; J. R. Banavar, F. Colaiori, A. Flammini, A. Giacometti, A. Maritan, A. Rinaldo, "Sculpting of a fractal river basin", Phys. Rev. Lett., 78:23 (1997), $4522-4525$.

[11] E. Somfai, L. M. Sander, "Scaling and river networks: a Landau theory for erosion", Phys. Rev. E, 56:1 (1997), R5-R8.

[12] S. Kramer, M. Marder, "Evolution of river networks", Phys. Rev. Lett., 68:2 (1992), 205-208.

[13] D. Sornette, Y.-C. Zhang, "Non-linear Langevin model of geomorphic erosion processes", Geophys. J. Internat., 113:2 (1993), 382-386.

[14] R. Pastor-Satorras, D. H. Rothman, "Stochastic equation for the erosion of inclined topography", Phys. Rev. Lett., 80:19 (1998), 4349-4352.

[15] R. Pastor-Satorras, D. H. Rothman, "Scaling of a slope: the erosion of tilted landscapes", J. Stat. Phys., 93:3-4 (1998), 477-500.

[16] P. S. Dodds, D. H. Rothman, "Scaling, universality, and geomorphology", Annu. Rev. Earth Planet Sci., 28 (2000), 571-610.

[17] A. Giacometti, "Local minimal energy landscapes in river networks", Phys. Rev. E, 62:5 (2000), 6042-6051.

[18] K. K. Chan, D. H. Rothman, "Coupled length scales in eroding landscapes", Phys. Rev. E, 63:5 (2001), 055102, 4 pp.

[19] M. Kardar, G. Parisi, Y.-C. Zhang, "Dynamic scaling of growing interfaces", Phys. Rev. Lett., 56:9 (1986), 889-892.

[20] H. Jeong, B. Kahng, D. Kim, "Anisotropic surface growth model in disordered media", Phys. Rev. Lett., 77:25 (1996), 5094-5097; H.-J. Kim, I.-M. Kim, J. M. Kim, "Hybridized discrete model for the anisotropic Kardar-Parisi-Zhang equation", Phys. Rev. E, 58:1 (1998), 1144-1147.

[21] E. Vivo, M. Nicoli, R. Cuerno, "Strong anisotropy in two-dimensional surfaces with generic scale invariance: Gaussian and related models", Phys. Rev. E, 86:5 (2012), 051611, 12 pp.; "Strong anisotropy in two-dimensional surfaces with generic scale invariance: Nonlinear effects", Phys. Rev. E, 89:4 (2014), 042407, 21 pp.; E. Vivo, M. Nicoli, M. Engler, T. Michely, L. Vázquez, R. Cuerno, "Strong anisotropy in surface kinetic roughening: Analysis and experiments", Phys. Rev. B, 86:24 (2012), 245427, 8 pp.

[22] Н. В. Антонов, П. И. Какинь, "Случайный рост границы раздела фаз в случайной среде: ренормгрупповой анализ простой модели", ТМФ, 185:1 (2015), 37-56.

[23] T. Hwa, M. Kardar, "Dissipative transport in open systems: an investigation of self-organized criticality", Phys. Rev. Lett., 62:16 (1989), 1813-1816; "Avalanches, hydrodynamics, and discharge events in models of sandpiles", Phys. Rev. A, 45:10 (1992), 7002-7023.

[24] B. Tadić, "Disorder-induced critical behavior in driven diffusive systems", Phys. Rev. E, $\mathbf{5 8 : 1}$ (1998), 168-173.

[25] N. V. Antonov, P. I. Kakin, "Effects of random environment on a self-organized critical system: renormalization group analysis of a continuous model", Eur. Phys. J: Web Conf., 108 (2016), 02009, 6 pp.

[26] С. И. Павлик, "Скейлинг для растущей границы раздела с нелинейной диффузией”, ЖЭТФ, 106:2 (1994), 553-559.

[27] Н. В. Антонов, Ал. Н. Васильев, "Квантово-полевая ренормгруппа в задаче о растущей границе раздела", ЖЭТФ, 108:3 (1995), 885-893. 
[28] Н. В. Антонов, "Ренормализационная группа в задаче о турбулентной конвекции пассивной примеси в случае нелинейной диффузии”, ЖЖТФ, 112:5(11) (1997), 1649-1663.

[29] N. V. Antonov, Nuclear and Particle Physics. Theoretical Physics, Proceedings of the XLVII Winter School of PNPI NRC KI (25 February - 2 March, 2013), ed. V. T. Kim, St. Petersburg Nucl. Phys. Inst., St. Petersburg, 2014, 147.

[30] J. Zinn-Justin, Quantum Field Theory and Critical Phenomena, International Series of Monographs on Physics, 77, Clarendon Press, Oxford, 1989.

[31] А. Н. Васильев, Квантовополевая ренормгруппа в теории критического поведения и стохастической динамике, Изд-во ПИЯФ, СПб., 1998.

[32] А.Н. Васильев, Функииональные методы в квантовой теории поля и статистике, ЛГУ, Л., 1976.

[33] Д. И. Казаков, “Об одном обобщении уравнений ренормгруппы для квантово-полевых теорий произвольного вида", ТМФ, 75:1 (1988), 157-160. 